\title{
Review of: "Efficacy of the ChAdOx1 nCoV-19 Covid- 19 Vaccine against the B.1.351 Variant"
}

\author{
Naeem Khan
}

Potential competing interests: The author(s) declared that no potential competing interests exist.

This is a potentially interesting article. On the positive side, there may be interest in the likely significant biological relevance of this Adenovirus Vector-based vaccine against SARS-CoV-2 against B.1.351 variant in terms of the immunological efficacy. In this paper authors did not explain why this vaccine is ineffective against B.1.351 variant. Different route of administration or potentially new vectors would might improve the efficacy against B.1.351 variant or possible new variants. 